\title{
Lévy Random Walks as Nonlinear Brownian Motion
}

\author{
Ihor Lubashevsky ${ }^{\dagger}$, Kimiaki Saitô $\hat{o}^{\ddagger}$, Yoshinori Uchimura ${ }^{\S}$ \\ ${ }^{\dagger}$ University of Aizu, Tsuruga, Ikki-machi, Aizu-Wakamatsu City, Fukushima 965-8580, Japan \\ ¥,§ Department of Mathematics, Meijo University, Tenpaku, Nagoya City, Aichi 468-8502, Japan \\ E-mail: † i-lubash@u-aizu.ac.jp, $\quad{ }^{\ddagger}$ ksaito@meijo-u.ac.jp, $\quad \S$ m0951501@ccalumni.meijo-u.ac.jp
}

\begin{abstract}
For describing irregular movement of animals in random search as well as patterns of human travel during daily activity an original model of Brownian nonlinear motion is proposed. The wandering particle is represented as a point in the extended phase space comprising its position, velocity, and, in addition, the acceleration. The acceleration is assumed to be governed by a certain random process with stochastic selfacceleration. The acceleration dynamics is described by a nonlinear stochastic differential equation of the Hänggi-Klimontovich type. Its regular component represents the preference of the particle moving with a certain fixed velocity. The stochastic component with the noise intensity growing with the acceleration is related to the active behavior of the wandering particle in changing the motion direction. The model is studied numerically. The obtained results allow us to state that the developed model generates motion trajectories that can be treated as Lévy random walks. The latter statement can be regarded as the main original point of the present work demonstrating a new approach to modeling the random searching based on continuous Markovian processes.
\end{abstract}

\section{Introduction}

During the last decades the notion of Lévy random walks has been developed to describe, in particular, irregular movement of animals in searching for food resources, mates, den sites, etc. as well as the trajectories that humans follow during their daily activity (for a review see, e.g. [1]). Such random walks can be treated as a collection of relatively straight fragments oriented randomly in space and separated by sharp turns. The characteristic feature of these straight fragments is the power-law distribution of their lengths. In the case at hand the velocity of the corresponding wandering "particle" does not exhibit strong variations so this powerlaw distribution is due to heavy tails in the distribution of the time elapsing between successive turns.

In spite of the achieved progress in modeling animal movement, there are a number of challenging problems in bridging the gap between behavioral ecology and statistical physics. Let us note two of them.

First, it is the optimal discretization of animal tra- jectories reproducing their basic properties with maximum fidelity. In particular, this poses a question as to whether the relationship between the statistics of move lengths and turning angles comes from the real behavioral events rather than the artificial discretization $[2,3]$.

Second, by now it has become evident that the movement patterns of many animals are of fractal, i.e., scalefree structure at least within multiple scales. These patterns can be described in terms of Lévy random walks or truncated ones (see, e.g., [1]). However the specific mechanisms by which such patterns arise are far from being understood well. There are different views on this subject. One of them is that the complexity of movement patterns is due to combination of various "behavioral modes" that change one another over time, e.g., it is the alternation of "encamped" and "exploratory" movements; a sophisticated implementation of such models is related to the concept of behavioral intermittence, for a detailed discussion and generalization see [2]. It assumes the animal movement to be made up of the locally static or diffusive search phases alternated with ballistic relocation during which detection is switched off. In this context the emergence of scale-free movement patterns should be a result of the animal adaptation. The mathematical description of this process is, however, far from being developed well. The matter is that the relevant governing equations have to deal with variables directly quantifying the animal behavior rather than the movement pattern. In the present work we consider a continuous Markovian random process whose characteristics mimic the main features of the animal behavior in search movement. The derived random walks seem to be able to exhibit the Lévy type statistics. Coupling these characteristics with the resource distribution, the landscape, the presence of other organisms, etc. this approach could enable one to describe complex phenomena observed in animal and human travel.

\section{Model Background}

To be specific in the following constructions we will keep in mind animals that under the normal conditions prefer to move with a speed about a certain value. For example, most sharks need to constantly swim in order 


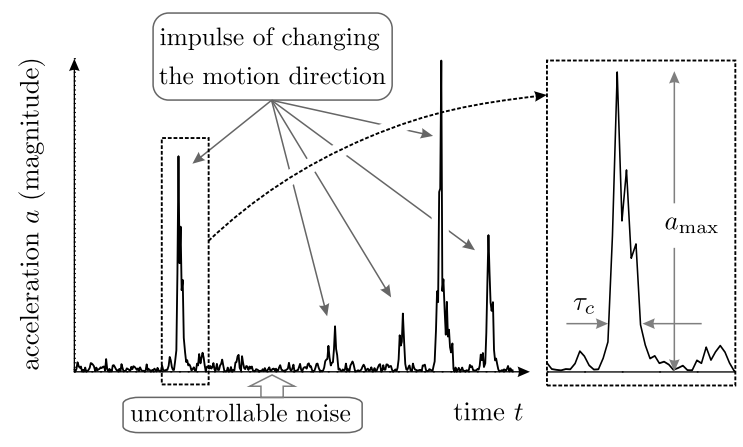

Fig. 1: Illustration of the acceleration time pattern characterizing the animal motion during the random search. In inset the characteristic form of control impulse is depicted.

to breath or not to sink because of their physiology. Birds usually cannot hover. In this case the control over the animal movement is reduced to changing or maintaining the direction of motion. In other words, on one hand, the acceleration $a$ is the control parameter via which an organism directly governs its motion. To change the motion direction the acceleration should take a certain value during a relatively short time interval $\tau_{c}$. On the other hand, this action requires some efforts on its own. So in describing the animal behavior the acceleration $a$ becomes an individual phase variable and a relevant governing equation has to determine its dynamics. For a detailed discussion of this aspect with respect to car driving a reader is referred to $[4,5]$.

Therefore, the action of an organism in governing its motion can be represented as a collection of spikes in the time pattern of the acceleration $a(t)$ illustrated in Fig. 1. Naturally, this pattern contains also small uncontrollable noise caused, for instance, by wind fluctuations when birds fly. A single action of an organism in governing its motion is quantified by a control impulse $\delta v$ estimated as the product of the acceleration maximum $a_{\max }$ times the duration $\tau_{c}$ of the spike, $\delta v \sim a_{\max } \tau_{c}$. It should be noted that the duration of single control impulse can be also treated as the correlation time interval of the $a(t)$-pattern.

In the frameworks of our approach the complexity of animal movement patterns is due to the control impulses varying in magnitude within an wide interval. In addition we will adopt the following two assumptions. First, the multitude of the control impulses $\{\delta v\}$ is mainly caused by variations in the acceleration maximum of individual spikes rather than changes of their duration. In other words, sharp turns in the animal movement are due to intensive actions of changing the motion direction rather then weak actions during long time intervals. Second, the basic properties of random search are caused by the stochastic factors in animal behavior. In mathematical terms, they should come from the characteristics of the stochastic component in the corresponding stochastic differential equation. Its regular component, roughly speaking, determines mainly the duration of individual actions in changing the motion direction. The purpose of the present work is to construct a nonlinear stochastic process that dealing with the particle acceleration a can reproduce the desired properties of random search.

The model to be developed inherits the main ideas of the generalized Cauchy stochastic processes which are known to exhibit the required properties and to generate time patterns like one shown in Fig. 1 (see, e.g., [6]-[11]). The key point of these Cauchy type models is stochastic differential equations similar to the following

$$
\frac{d a}{d t}=-\lambda a+\sqrt{\frac{1}{\tau}}\left[a_{0} \xi_{\mathrm{a}}(t)+a * \xi_{\mathrm{m}}(t)\right]
$$

or equivalent one

$$
\frac{d a}{d t}=-\lambda a+\sqrt{\frac{1}{\tau}}\left[a_{0}^{2}+a^{2}\right]^{1 / 2} * \xi(t) .
$$

Here the pair $\left\{\xi_{\mathrm{a}}(t), \xi_{\mathrm{m}}(t)\right\}$ as well as $\xi(t)$ are independent realizations of white noise of unit amplitude, the parameter $\tau$ with units of time directly specifies the intensity of the multiplicative noise, the parameter $a_{0}$ with units of acceleration together with $\tau$ determines the intensity of the additive noise, and the quantity $\lambda$ is the rate of the regular dissipation of the variable $a$. The asterisk in equations (1) indicates that they are written in the Hänggi-Klimontovich form. It should be noted that when $\lambda \tau \sim 1$ the time scale $\tau$ characterizes correlations in time variations of the quantity $a$. The equivalence of equations (1) stems from both of them giving rise to the same Fokker-Planck equation for the probability distribution density $P(a, t)$

$$
\frac{\partial P}{\partial t}=\frac{\partial}{\partial a}\left[g^{2}(a) \frac{\partial P}{\partial a}+\lambda a P\right]
$$

with the function $g(a)$ given by the expression

$$
g(a)=\left[\frac{1}{2 \tau_{m}}\left(a_{0}^{2}+a^{2}\right)\right]^{1 / 2} .
$$

As far as modeling animal movements is concerned, the time scale $\tau$ may be treated as the characteristic duration of single actions in governing the motion direction. An equation similar to (1b) also may be used where, however, expression (3) describing the intensity of nonlinear noise has to be modified in order to take into account the bounded capacity of animal actions. To allow for the latter factor the ansatz

$$
g(a)=\frac{a_{s}}{\sqrt{2 \tau}}\left[\frac{\Delta^{2}+\left(a / a_{s}\right)^{2}}{1+\left(a / a_{s}\right)^{2}}\right]^{1 / 2} .
$$

is employed. Its structure stems from the expected dependence $g(a)$ shown in Fig. 2. Here the value 


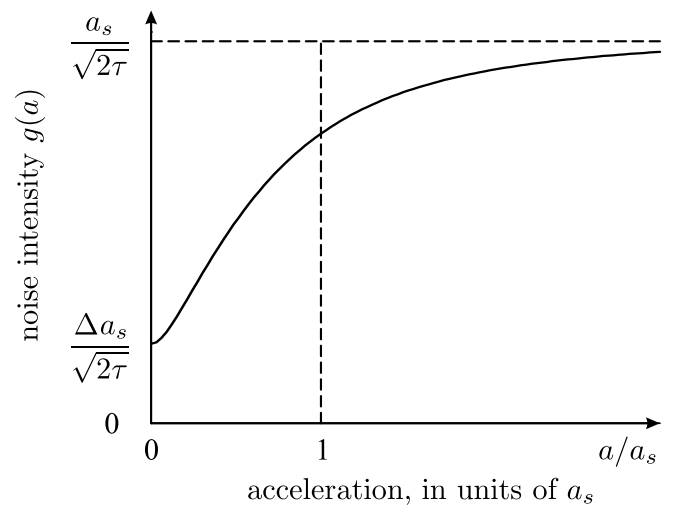

Fig. 2: The expected dependence of the Langevin force intensity $g(a)$ on the particle acceleration $a$. It has been used in constructing ansatz (4) entering the model for animal movement where the acceleration $a$ is treated as the control variable in governing the motion direction. The parameter $a_{s}$ characterizes the bounded capacity of animal actions.

$a_{s}$ estimates the characteristic accelerations attained during changing the motion direction in the diffusive search phase. The acceleration $\Delta a_{s}$ with the coefficient $0<\Delta<1$ quantifies the intensity of turning during the animal relocation as well as takes into account the effect of uncontrollable noise.

The form of equations (1) is invariant with respect to converting from the Hänggi-Klimontovich type to other types of stochastic differential equations within the renormalization of the constant $\lambda$. This invariance, however, does not hold when the intensity of the Langevin random forces in equation (1b) is determined by expression (4). So in further constructions we should specify the type of stochastic differential equations to be used at the initial stage. When an organism is searching for certain objects it is natural to suppose that a given local action of changing the motion direction is caused by a local target, visible or expected one. This target has to be a terminal point of the local fragment of the animal trajectory. It matches the concept of postpoints in constructing stochastic differential equations $[12,13]$. As a result, stochastic differential equations of the Hänggi-Klimontovich type will be used in describing the animal movement (for details see Appendix A). In particular, equation (1b) with ansatz (4) will be the key point in constructing the model.

In this case the value $a_{s}$ can be also treated qualitatively as the upper boundary of accelerations taking place in the animal movements. The parameter $\Delta$ quantifies the preference of the organism to make rare sharp turns in changing the motion direction rather than to veer gradually. The first statement stems directly from the form of the stationary distribution $P_{\mathrm{st}}(a)$ of the acceleration, which in the given case by virtue of (2) and (4) is written as (see Appendix B)

$$
P_{\mathrm{st}}(a) \propto \frac{e^{-\lambda \tau\left(a / a_{s}\right)^{2}}}{\left[1+\left[a /\left(\Delta a_{s}\right)\right]^{2}\right]^{\lambda \tau\left(1-\Delta^{2}\right)}} .
$$

So when $\Delta \ll 1$ the acceleration $a$ is distributed inside the interval $\Delta a_{s} \lesssim a \lesssim a_{s}$ according to the powerlaw and then its distribution is actually cut off by the Gaussian cofactor for $a \gtrsim a_{s}$. The second statement is justified by the results to be described in Sec. 4 .

In principle, similar statistical properties are exhibited by random walks governed equations (1) where the regular linear term is added with a cubic nonlinearity [14]. However, first, similar time patterns of $a(t)$ seem to be generated by the stochastic differential equations where either $(i)$ the regular term contains a cubic nonlinearity and the Langevin force is composed of the additive and multiplicative noise or (ii) the regular term contains only the linear force whereas the intensity of the Langevin force is given by ansatz (4). This equivalence holds, at least, in the one-dimensional case [11] (see also Appendix A). Second, within the former approach the cubic nonlinearity has to be attributed to the physical ability of an organism to accelerate with high intensity. In contrast, within the latter approach the acceleration cutoff is mainly due to the behavioral stimuli. Therefore in the present work we confine our constructions to the latter one.

\section{Model}

The mathematical background presented in Sec. 2 prompts us to imitate the animal movements discussed in Introduction by random walks of a Brownian particle governed by two factors. The first one is its preference to move at a certain predetermined velocity $v_{0}$. It will be described within the Rayleigh-Helmholtz model widely used in simulating active Brownian motion (see, e.g., $[15,16])$. In terms of social forces [17] it regards this velocity preference as an effective force

$$
\mathbf{F}_{\mathrm{vp}}(v)=\varpi\left(1-\frac{\mathbf{v}^{2}}{v_{0}^{2}}\right) \mathbf{v}
$$

depending on the current velocity $\mathbf{v}$ in such a manner that the point $|\mathbf{v}|=v_{0}$ be stable. In expression (6) $\varpi$ is a certain coefficient with units of reciprocal time. The second factor is the active behavior of the Brownian particle in controlling the motion direction described as an additional component a governed by a stochastic differential equation similar to (1b) with the intensity of the Langevin forces determined by expression (4).

Namely the system under consideration is specified by the following coupled equations for the velocity $\mathbf{v}=$ 


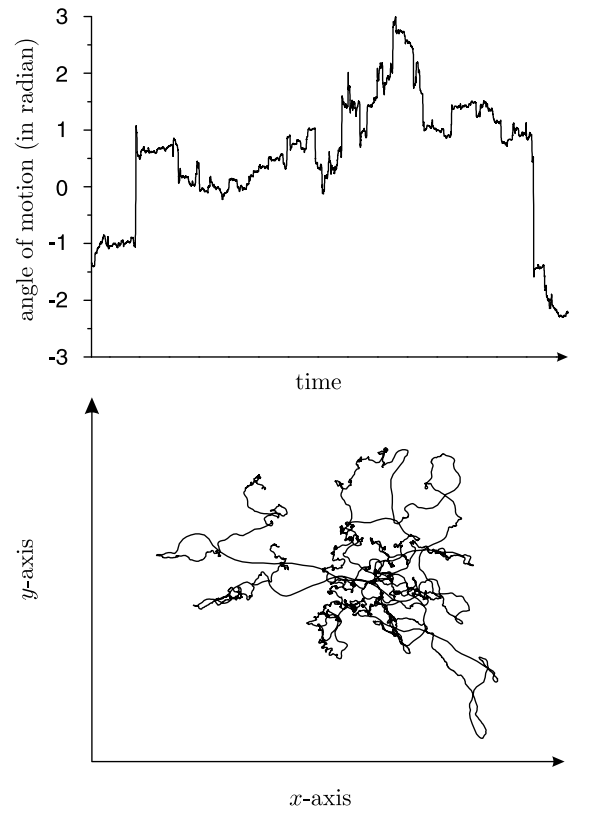

Fig. 3: The characteristic form of the time pattern $\{\phi(t)\}$ of the angle between the particle velocity and the $x$-axis as well as the corresponding trajectory of the animal motion governed by the model under consideration.

$d \mathbf{r} / d t$ of the given particle and its active behavior

$$
\begin{aligned}
& \frac{d \mathbf{v}}{d t}=\varpi\left(1-\frac{\mathbf{v}^{2}}{v_{0}^{2}}\right) \mathbf{v}+\mathbf{a}, \\
& \frac{d \mathbf{a}}{d t}=-\frac{\lambda}{\tau} \mathbf{a}+\frac{\sqrt{2} a_{s}}{\sqrt{\tau}} \sqrt{\frac{\Delta^{2} a_{s}^{2}+\mathbf{a}^{2}}{a_{s}^{2}+\mathbf{a}^{2}}} * \boldsymbol{\xi}(\boldsymbol{t}) .
\end{aligned}
$$

Here we have used the same notions as in Introduction and, in addition, the introduced vectors are assumed to belong to the two-dimensional Euclidean space, $\mathbf{r}=$ $\{x, y\}, \mathbf{v}, \mathbf{a}, \boldsymbol{\xi} \in \mathbb{R}^{2}$, with the components $\left\{\xi_{1}(t), \xi_{2}(t)\right\}$ of the random vector $\boldsymbol{\xi}(t)$ being independent realizations of white noise with unit amplitude, i.e., for $i=1,2$

$$
\left\langle\xi_{i}(t)\right\rangle=0, \quad\left\langle\xi_{i}(t) \xi_{i^{\prime}}\left(t^{\prime}\right)\right\rangle=\delta_{i i^{\prime}} \delta\left(t-t^{\prime}\right),
$$

where $\delta\left(t-t^{\prime}\right)$ is the Dirac $\delta$-function, $\delta_{i i^{\prime}}$ is the Kronecker symbol, and the symbol $\langle\ldots\rangle$ denotes averaging over possible realizations of the noise components.

\section{Results of Simulation. Conclusion}

The system of equations (7), (8) was solved using the stochastic Runge-Kutta method RI1 approximating multidimensional Wiener process Itô equations with weak order $(3,2)$ [18]. Naturally, in numerical simulation the given system was reduced to the Itô form using the technique described in Appendix A. Here only the preliminary results are demonstrated which, nevertheless, enables us to draw the main conclusion about the developed model. A thorough analysis is the subject of following publications.

Figure 3 depicts, first, the obtained time pattern $\{\phi(t)\}$ of the angle $\phi$ formed by the particle velocity $\mathbf{v}$ and the $x$-axis. As seen, the animal motion described within the given approach does undergo a variety of sharp turns. Moreover, appealing to the results of $[8,9]$ it is reasonable to expect that the random variable $a$ as well as the turn angles should obey statistics of truncated Lévy random walks with the cutoff about $a_{s}$. Assuming quantities such as the values $\lambda$ and $\Delta$ to depend on the position $\mathbf{r}$ of a given organism in space and its velocity $\mathbf{v}$ as well as the same collection of variables of other organisms one can couple the animal behavior with the state of environment.

Second, the corresponding trajectory of the particle motion in the space $\{x, y\}$ (Fig. 3) also looks like a collection of relatively straight fragments separated by sharp turns corresponding to jumps in the pattern $\{\phi(t)\}$. Nevertheless the obtained form of the trajectory allows us to pose a question about constructing an optimal partition exhibiting the real properties of such random motion. It could be done in the following way. The discrete representation of the generated random walks is constructed via the introduction of a certain threshold $\phi_{\mathrm{th}}$ in the variations of the motion angle $\phi(t)$. When the current angle $\phi(t)$ exceeds the angle $\phi_{i-1}$ fixed at the previous moment of time $t_{i-1}$ by the threshold $\phi_{\mathrm{th}}$ the given time moment $t_{i}$ is also fixed and the fragment of the particle motion during the time interval $\left(t_{i-1}, t_{i}\right)$ is replaced by the straight line connecting the particle positions at $t_{i-1}$ and $t_{i}$. Repeating the given procedure we construct the standard representation of Lévy random walks. In particular, changing the magnitude of the threshold $\phi_{\text {th }}$ we obtain the collection of different discrete representations of such random walks that should be treated as equivalent.

Finally, it is necessary to point out that the inclusion of the particle acceleration a into the collection of the phase variables in addition to the spatial position $\mathbf{r}$ and velocity $\mathbf{v}$ has enabled us to describe the active behavior, e.g., of an animal in searching for food resources governed also by its internal stimuli. So the given model does not meet the paradigms of Newtonian mechanics and can be regarded as a new type of mathematical problems describing animate beings. Besides, the dependence of the noise intensity on the acceleration a allows for the behavioral intermittence caused by the animal alternately switching between "encamped" and "exploratory" movements in search strategy.

\section{Acknowledgments}

The work was supported in part by the JSPS Grant 245404100001 ("Grants-in-Aid for Scientific Research" Program) as well as the Competitive Research Funding of the University of Aizu, Project P-25, FY2012. 


\section{A Equivalent forms of stochastic differential equations}

Let us consider dynamics of stochastic variable $\mathbf{a}=$ $\left\{a_{1}, a_{2}, \ldots, a_{N}\right\} \in \mathbb{R}^{N}$ belonging to the Euclidean $N$ dimensional space governed by the following stochastic differential equation

$$
d a_{i}=\mathcal{F}_{i}^{(\theta)}(\mathbf{a}) d t+\mathcal{G}(\mathbf{a}+\theta d \mathbf{a}) d W_{i}
$$

Here the collection of functions $\left\{\mathcal{F}_{i}^{(\theta)}(\mathbf{a})\right\}$ forms the vector $\mathbf{F}^{(\theta)}(\mathbf{a})$ of the $\mathbb{R}^{N}$-space, the scalar function $\mathcal{G}(\mathbf{a})=\mathcal{G}(|\mathbf{a}|)$ is assumed to depend only on the magnitude $a:=|\mathbf{a}|$ of the vector $\mathbf{a}$, the collection $\{W(t)\}$ is made of $N$ independent realizations of the Wiener process with unit amplitude, i.e.,

$$
\left\langle d W_{i}\right\rangle=0, \quad\left\langle d W_{i} d W_{j}\right\rangle=\delta_{i j} d t,
$$

where, as before, $\langle\ldots\rangle$ designates the averaging over the realizations of the given Wiener processes and $\delta_{i j}$ is the Kronecker delta. By virtue of (11) the quantity $d W_{i}$ may be treated as a variable of order $(d t)^{1 / 2}$. The parameter $\theta \in[0,1]$ specifies the point $\mathbf{a}_{\theta}:=\mathbf{a}+\theta d \mathbf{a}$ at which the intensity $\mathcal{G}(a)$ of the Langevin random forces is taken at the step from $\mathbf{a}$ to $\mathbf{a}+d \mathbf{a}$. It is worthy to be noted that, first, the parameter $\theta$ is an individual characteristics of a given stochastic process which together with the rate $\mathbf{F}^{(\theta)}(\mathbf{a})$ of the regular drift and the intensity $\mathcal{G}(a)$ of the Langevin forces makes up the description of the stochastic process under consideration. Second, the value $\theta=0$ matches the Îto type of stochastic processes, $\theta=1 / 2$ is related to the Stratonovich processes, and in the given case of the isotropic Langevin forces the value $\theta=1$ corresponds to the Hänggi-Klimontovich processes. In principle, the regular drift $\mathbf{F}^{(\theta)}(\mathbf{a})$ should be also taken at the point $\mathbf{a}_{\theta}$, however, the resulting difference is of order of $(d t)^{3 / 2}$ and, thus, can be ignored.

We regard the given equation (10) as a stochastic differential equation of the $\theta$-type. It can be converted into the other types in such a manner that the resulting stochastic differential equations produce the same Fokker-Plank equation. In this sense all of them will be treated as equivalent. In order to construct the equivalent stochastic process of the Hänggi-Klimontovich type we expand the function $\mathcal{G}(\mathbf{a}+\theta d \mathbf{a})$ into the Taylor series with respect to the difference $d \mathbf{a}$ and keep up only the essential terms, namely, the terms of order 0 and $1 / 2$ with respect to the time difference $d t$. In this way we get

$$
\mathcal{G}(\mathbf{a}+\theta d \mathbf{a})=\mathcal{G}(a)+\theta \sum_{j=1}^{N} \frac{\partial \mathcal{G}(a)}{\partial a_{j}} d a_{j} .
$$

As stems from (10)

$$
d a_{i}=\mathcal{G}(a) d W_{i}
$$

by the order of $1 / 2$ in $d t$. Thereby we may write

$$
\mathcal{G}(\mathbf{a}+\theta d \mathbf{a})=\mathcal{G}(a)+\frac{\theta}{2} \sum_{j=1}^{N} \frac{\partial \mathcal{G}^{2}(a)}{\partial a_{j}} d W_{j} .
$$

The substitution of (14) into (10) yields the desired stochastic differential equation of the Itto type

$$
d a_{i}=\mathcal{F}_{i}^{(0)}(\mathbf{a}) d t+\mathcal{G}(a) d W_{i},
$$

where the rate $\mathcal{F}_{i}^{(0)}(\mathbf{a})$ of the Ito regular drift is

$$
\mathcal{F}_{i}^{(0)}(\mathbf{a})=\mathcal{F}_{i}^{(\theta)}(\mathbf{a})+\frac{\theta}{2} \frac{\partial \mathcal{G}^{2}(a)}{\partial a_{i}} .
$$

In deriving (15) the replacement

$$
d W_{i} d W_{j} \rightarrow\left\langle d W_{i} d W_{j}\right\rangle
$$

holding within the given equivalence and, then, equalities (11) have been employed.

The family of equations (10) related to each other via expression (16) for various values of the parameter $\theta$ is considered to be the equivalent description of the same stochastic process.

The probability distribution function $P(\mathbf{a}, t)$ describing the stochastic process governed by equation (15) obeys the following Fokker-Planck equation

$$
\frac{\partial P}{\partial t}=\sum_{j=1}^{N} \frac{\partial}{\partial a_{j}}\left[\frac{\partial}{\partial a_{j}}\left(\frac{1}{2} \mathcal{G}^{2}(a) P\right)-\mathcal{F}_{i}^{(0)}(\mathbf{a}) P\right] .
$$

In particular, for $\theta=1$ we get

$$
\frac{\partial P}{\partial t}=\sum_{j=1}^{N} \frac{\partial}{\partial a_{j}}\left[\frac{1}{2} \mathcal{G}^{2}(a) \frac{\partial P}{\partial a_{j}}-\mathcal{F}_{i}^{(1)}(\mathbf{a}) P\right] .
$$

by virtue of (16). Form (18) of the Fokker-Planck equation is actually the key point of the HänggiKlimontovich type of stochastic processes and, thereby, in the isotropic case of the random Langevin forces the $(\theta=1)$-type processes do belong to the HänggiKlimontovich type. It should be note that for the general multidimensional processes the latter statement does not hold.

When the rate $\mathbf{F}^{(1)}(\mathbf{a})$ of the Itto regular drift is potential with isotropic symmetry, i.e., there is a function $\mathcal{U}(a)$ such that

$$
\mathcal{F}_{i}^{(1)}(\mathbf{a})=-\frac{\partial \mathcal{U}(a)}{\partial a_{i}},
$$

equation (18) admits the stationary solution $P_{\mathrm{st}}(a)$ meeting the zero probability flux

$$
\frac{1}{2} \mathcal{G}^{2}(a) \frac{\partial P_{\mathrm{st}}}{\partial a_{j}}-\mathcal{F}_{i}^{(1)}(\mathbf{a}) P_{\mathrm{st}}=0
$$

and, as a result, specified by the expression

$$
P_{\mathrm{st}}(a)=\frac{1}{Z} \exp \left\{-\int_{0}^{a} \frac{2}{\mathcal{G}^{2}\left(a^{\prime}\right)} \frac{d U\left(a^{\prime}\right)}{d a^{\prime}} d a^{\prime}\right\},
$$

where $Z$ is the partitioning coefficient. 


\section{B Canonical form of the generalized Cauchy process}

Keeping in mind the notations used previously we consider the following stochastic differential equation of the Hänggi-Klimontovich type

$$
d a=-F(a) d t+G(a) * d W
$$

where $W(t)$ is the Wiener process and the functions $F(a), G(a)$ are specified either by the expressions

$$
\begin{aligned}
F_{1}(a) & =\lambda a, \\
G_{1}(a) & =\sqrt{\frac{a_{s}^{2}}{\tau}}\left[\frac{\Delta^{2}+\left(a / a_{s}\right)^{2}}{1+\left(a / a_{s}\right)^{2}}\right]^{1 / 2}
\end{aligned}
$$

or by the expressions

$$
\begin{aligned}
& F_{2}(a)=\lambda a\left[1+\left(a / a_{s}\right)^{2}\right] \\
& G_{2}(a)=\sqrt{\frac{a_{s}^{2}}{\tau}}\left[\Delta^{2}+\left(a / a_{s}\right)^{2}\right]^{1 / 2} .
\end{aligned}
$$

As follows from (22) the probability distribution function $P(a, t)$ obeys the Fokker-Planck equation

$$
\frac{\partial P}{\partial t}=\frac{\partial}{\partial a}\left[\frac{G^{2}(a)}{2} \frac{\partial P}{\partial a}+F(a) P\right]
$$

and its steady state solution is

$$
P_{\mathrm{st}}(a) \propto \exp \left[-\int_{0}^{a} \frac{2 F\left(a^{\prime}\right)}{G^{2}\left(a^{\prime}\right)} d a^{\prime}\right] .
$$

In particular, in both the cases the stationary distribution $P_{\text {st }}(a)$ has the same form

$$
P_{\mathrm{st}}^{(1,2)}(a) \propto \frac{e^{-\lambda \tau\left(a / a_{s}\right)^{2}}}{\left[1+\left[a /\left(\Delta a_{s}\right)\right]^{2}\right]^{\lambda \tau\left(1-\Delta^{2}\right)}} .
$$

\section{References}

[1] O. Bénichou, C. Loverdo, M. Moreau, and R. Voituriez, Intermittent search strategies, Rev. Mod. Phys. 83, pp. 81-129 (2011).

[2] F. Bartumeus, Behavioral intermittence, Lévy patterns, and randomness in animal movement, Oikos 118, pp. 488-494 (2009).

[3] P. Turchin, Quantitative Analysis of Movement: measuring and modeling population redistribution in plants and animals (Sinauer Associates, Sunderland, MA, 1998).

[4] I. Lubashevsky, P. Wagner, and R. Mahnke, Rational-driver approximation in car-following theory, Phys. Rev. E 68, p. 056109 (2003).
[5] I. Lubashevsky, P. Wagner, and R. Mahnke, Bounded rational driver model, Eur. Phys. J. B 32, pp. 243-247 (2003).

[6] H. Konno and F. Watanabe, Maximum likelihood estimators for generalized Cauchy processes, J. Math. Phys. 48, 103303 (2007).

[7] H. Sakaguchi, Fluctuation dissipation relation for a Langevin model with multiplicative noise, J. Phys. Soc. Japan 70, pp. 3247-3250 (2001).

[8] I. Lubashevsky, R. Friedrich, and A. Heuer, Realization of Lévy walks as Markovian stochastic processes, Phys. Rev. E 79, p. 011110 (2009).

[9] I. Lubashevsky, R. Friedrich, and A. Heuer, Continuous-time multidimensional Markovian description of Lévy walks, Phys. Rev. E 80, p. 031148 (2009).

[10] I. Lubashevsky, A. Heuer, R. Friedrich, R. Usmanov, Continuous Markovian model for Lévy random walks with superdiffusive and superballistic regimes, Eur. Phys. J. B 78, pp. 207-216 (2010).

[11] I. A. Lubashevsky, Truncated Lévy flights and generalized Cauchy processes, Eur. Phys. J. B 82, pp. 189-195 (2011).

[12] T. Morita, H. Hara, Solution of the Fokker-Planck equation with spatial coordinate-dependent moments, Physica A 101, pp. 283-288 (1980).

[13] T. Morita, H. Hara, Solution of the Fokker-Planck equation with spatial coordinate-dependent moments in many dimensions, Physica A $\mathbf{1 2 5}$, pp. 607-618 (1984)

[14] H. Konno, Y. Tamura, A generalized Cauchy process having cubic nonlinearity, Rep. Math. Phys. 67, pp. 179-195 (2011).

[15] Yu. L. Klimontovich, Statistical Theory of Open Systems, Kluwer Academic Publishers, Dordrecht, 1995.

[16] F. Schweitzer, Brownian Agents and Active Particles, Springer-Verlag, Berlin, 2003.

[17] D. Helbing, Traffic and related self-driven manyparticle systems, Rev. Mod. Phys. 73, pp. 10671141 (2001).

[18] A. Rößler, Second order Runge-Kutta methods for Itô stochastic differential equations, SIAM J. Numer. Anal. 47, pp. 1713-1738 (2009). 